Published in final edited form as:

J Geriatr Oncol. 2013 October ; 4(4): 353-361. doi:10.1016/j.jgo.2013.05.004.

\title{
A planned, prospective comparison of short-term quality of life outcomes among older breast cancer patients treated with standard chemotherapy in a randomized clinical trial vs. an observational study: CALGB \#49907 and \#369901 is
}

\author{
Jeanne S. Mandelblatt ${ }^{a},{ }^{*}$, Solomon B. Makgoeng ${ }^{\mathrm{a}}$, Gheorghe Luta ${ }^{\mathrm{b}}$, Arti Hurria ${ }^{\mathrm{c}}$, Gretchen \\ Kimmick $^{d}$, Claudine Isaacs ${ }^{e}$, Michelle Tallarico $^{a}$, William T. Barry ${ }^{\dagger}$, Brandy Pitcher $^{\dagger}$, Eric P. \\ Winerg $^{\mathrm{g}}$, Clifford Hudis ${ }^{\mathrm{h}}$, Harvey J. Cohen', and Hyman B. Muss ${ }^{\mathrm{j}}$ \\ aDepartment of Oncology, Georgetown University Medical Center and Lombardi Cancer Center, \\ Cancer Prevention and Control Program, Washington, DC, USA \\ bDepartment of Biostatistics, Bioinformatics and Biomathematics, Georgetown University Medical \\ Center and Lombardi Cancer Center, Washington, DC, USA
}

'Department of Medical Oncology \& Therapeutics Research, City of Hope, LA, CA, USA

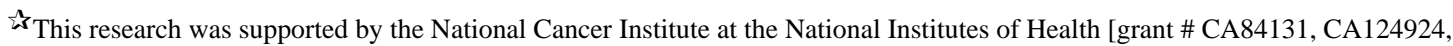
CA127617 and CA96940 to JSM] and by the National Institute on Aging [grant \# CA85850 to HM]. The research was also supported, in part, by the Clinical and Molecular Epidemiology (now NTSR) and Biostatistics and Bioinformatics Shared Resources at Lombardi Comprehensive Cancer Center funded by the National Cancer Institute at the National Institutes of Health [grant \# P30CA51008]. The research for CALGB 369901 and CALGB 49907 was also supported, in part, by the National Cancer Institute at the National Institutes of Health [grant \# CA31946 to the Cancer and Leukemia Group B (Monica Bernagnoli, MD, Chairman) and grant \# CA33601 to the CALGB Statistical Center (Stephen George, PhD)]. The content of this manuscript is solely the responsibility of the authors and does not represent the official views of the National Cancer Institute at the National Institutes of Health or the Cancer and Leukemia Group B. Cancer and Leukemia Group B is presently part of the Alliance for Clinical Trials in Oncology. These studies were also funded, in part, by a grant to support patient accrual from Amgen Pharmaceuticals to the CALGB Foundation.
\end{abstract}

() 2013 Published by Elsevier Ltd.

"Corresponding author at: Lombardi Comprehensive Cancer Center, 3300 Whitehaven Blvd, Suite 4100, Washington, DC 20007, USA. Tel: +1 202687 0812; fax: +1 202687 0305., mandelbj@ georgetown.edu (J.S. Mandelblatt).

Conflict Of Interest Declarations

Dr. Arti Hurria: Seattle Genetics, Amgen Pharmaceuticals and Genentech (Consultant); Glaxo Smith Kline, Abraxis Bioscience, and Celgene (Research Support).

Dr. Claudine Isaacs: Genentech and Glaxo Smith Kline (Consultant).

All remaining authors have declared no conflicts of interest.

Author Contributions

Jeanne S. Mandelblatt, MD, MPH: study conception and design, acquisition of subjects and data, analysis and interpretation of data, preparation of manuscript, and approval of final article.

Solomon B. Makgoeng, MS: analysis and interpretation of data, preparation of manuscript, approval of final article.

Gheorghe Luta, PhD: study concept and design; analysis and interpretation of data, preparation of manuscript, approval of final article. Arti Hurria, MD: acquisition of subjects and data, analysis and interpretation of data, preparation of manuscript, approval of final article.

Gretchen Kimmick, MD: acquisition of subjects and data, analysis and interpretation of data, preparation of manuscript, approval of final article.

Claudine Isaacs, MD: acquisition of subjects and data, preparation of manuscript, approval of final article.

Michelle Tallarico, MPH: acquisition of subjects and data, preparation of manuscript, approval of final article.

William T. Barry, PhD: analysis and interpretation of data, preparation of manuscript, approval of final article.

Brandy Pitcher, MS: acquisition of data, preparation of manuscript, approval of final article.

Eric P. Winer, MD: acquisition of subjects and data, preparation of manuscript, approval of final article.

Clifford Hudis, MD: acquisition of subjects and data, preparation of manuscript, approval of final article.

HarveyJ. Cohen, MD: study concept and design, acquisition of subjects and data, analysis and interpretation of data, preparation of manuscript, and approval of final article.

Hyman B. Muss, MD: study concept and design, acquisition of subjects and data, analysis and interpretation of data, preparation of manuscript, and approval of final article. 
${ }^{d}$ Department of Medicine, Division of Medical Oncology, Duke University Medical Center, Durham, NC, USA

eDepartment of Medicine, Georgetown University School of Medicine and Lombardi Cancer Center, Breast Cancer Program, Washington, DC, USA

fDepartment of Biostatistics and Bioinformatics, Duke University Medical Center, and Cancer and Leukemia Group B Statistical Center, Durham, NC, USA

gDepartment of Medical Oncology, Dana-Farber Cancer Institute, Boston, MA, USA

hDepartment of Medicine, Memorial Sloan-Kettering Cancer Center, New York, NY, USA

'Department of Medicine and Center for the Study of Aging and Human Development, Duke University, Durham, NC, USA

jDepartment of Medicine, University of North Carolina, Chapel Hill, NC, USA

\section{Abstract}

Objectives-Patients $\geq 65$ years old ("older") are often not included in randomized clinical trials (RCT), but when they are, care in an RCT might improve quality of life (QoL). We conducted a prospective comparison of QoL among older women receiving standard chemotherapy from the same cooperative group physicians in an RCT vs. an observational study ("off-trial").

Methods-Older women with invasive, non-metastatic breast cancer ( $\mathrm{n}=150 \mathrm{RCT}$; 530 offtrial) were included. Linear mixed-effects models tested associations between chemotherapy onvs. off-trial and changes in EORTC (European Organisation for Research and Treatment of Cancer Quality of Life Questionnaire) QoL scores over 24 months, controlling for pre-treatment QoL, age, education, tumor factors, comorbidity, and other covariates.

Results-Anthracycline regimens were used by $58 \%$ of women treated on-trial vs. $54 \%$ of those treated off-trial. Women in the RCT reported an adjusted mean increase of 13.7 points (95\% CI $10.2,17.1$ ) in global QoL at 24 months (vs. mid-treatment), while women treated off-trial had only an adjusted improvement of 7.0 points $(95 \%$ CI $3.5,10.4 ; \mathrm{p}=.007$ for difference in mean changes). Women in the RCT had significantly greater improvement in emotional function than those treated off-trial, controlling for baseline; they also had greater reductions in therapy side effects and fatigue at 24 months than women off-trial, controlling for covariates.

Conclusion-There may be different QoL trajectories for older women undergoing breast cancer chemotherapy on- vs. off-trial. If confirmed, the results suggest that the extra monitoring and communication within an RCT could provide the infrastructure for interventions to address symptoms and improve QoL for the growing older cancer population.

\section{Keywords}

Older patients; Breast cancer; Chemotherapy; Quality of Life; Randomized clinical trials; Observational studies

\section{Introduction}

Women 65 years and older, hereafter older, constitute almost half of all new invasive breast cancer cases and account for two-thirds of the deaths each year from this disease. ${ }^{1}$ With the graying of America, the absolute number of older breast cancer patients and survivors will increase substantially over the coming decades. Despite this demographic imperative, we know little about chemotherapy efficacy and post-chemotherapy quality of life (QoL) in 
older women since this group has been under-represented in randomized clinical trials (RCTs). ${ }^{2}$

Kemeny and colleagues reported that older women would enroll in a chemotherapy clinical trial if it was offered to them. ${ }^{3}$ Further, there are now trials designed specifically for the older cancer patients. ${ }^{4}$ Increasing the number of older women receiving chemotherapy within RCTs could increase the knowledge base about treatment efficacy and might have additional benefits for this group. For instance, the added attention and required monitoring in trial settings could potentially result in better outcomes for older women than those receiving comparable therapy delivered outside of trials.

We designed a prospective comparison of an RCT evaluating standard chemotherapy and oral capecitabine ${ }^{4}$ and an observational study of standard chemotherapy. ${ }^{5}$ The protocols for these studies had nearly identical inclusion criteria, were conducted in the same cooperative group sites by the same groups of providers and included a core of shared instruments. In this paper, we use data from women in the standard chemotherapy arm of the RCT and women receiving chemotherapy in the observational study to compare 24-month posttreatment QoL outcomes for these on- and off-RCT groups. We hypothesized that the women treated on the trial would have better QoL than women in the observational study even after considering baseline QoL, socio-demographic, clinical, and care setting differences between the groups. If there is improved QoL for women treated in clinical trials, this might suggest that there could be benefits from the enhanced surveillance for toxicity, attention to side effects and/or other interactions with providers associated with the quality and intensity of care delivered in RCTs. If confirmed, this information could advance knowledge to improve care of the growing population of older cancer patients.

\section{Methods}

The studies were conducted at 75 Cancer and Leukemia Group B (CALGB) sites (now part of the Alliance for Clinical Trials in Oncology). The protocols met HIPAA standards and were approved by the CALGB, the National Cancer Institute and all local IRBs. The studies and settings have been described in detail elsewhere ${ }^{4,5}$ and are summarized below. Protocol \#49907 was an RCT comparing oral capecitabine and standard chemotherapy in older women and included an embedded QoL study. ${ }^{6}$ The parallel observational study (protocol \#369901) evaluated factors associated with older women's decisions to receive chemotherapy and assessed QoL. ${ }^{5,7}$ Site clinical research associates obtained written consent from participants in both studies.

\subsection{Setting and Population}

Eligible participants in both protocols were 65 years or older, with newly diagnosed, primary histologically confirmed invasive non-metastatic breast cancer tumors stage T1-3, N0-3, M0. All performance status categories were eligible for the observational study. In the RCT women were required to have a performance status of 0-2 (fully active, or some impairments but can work, and ambulatory and can do self-care, but can't work, respectively) vs. 3-5 (confined, totally disabled, or dead, respectively) as measured by the treating physicians and expected survival greater than five years; performance status was not a criteria for the observational study and was not measured. For both studies, women with a score of $\geq 11$ on the Blessed Orientation-Memory-Concentration test ${ }^{8}$ were excluded as having potential cognitive impairment that might interfere with ability to consent and complete study procedures.

The RCT enrolled women in the QoL component from September 2001 to December 2006; follow-up was completed by 2008. The trial required six survey assessments over the course 
of 24 months and visits for RCT-specific laboratory assessments. The observational study enrolled women from December 2004 to April 2011. The observational study required four survey assessments during the first 24 months and no study-specific laboratory assessments. We considered year of enrollment in our statistical analyses.

For the analysis of QoL outcomes, the target population included women from the RCT on the standard chemotherapy arm and women from the observational study who received the same standard chemotherapies (Fig. 1). The mid-treatment assessment was the first timepoint assessed in the observational study. Therefore, for this analysis we included the 150 and 530 women who completed mid-treatment interviews in the RCT and observational study, respectively (Fig. 1). Note that none of the women in the observational study were participants in other treatment trials. Women in the target population for the observational study tended to be older than those who were included in the RCT for the QoL analysis.

\subsection{Data Collection}

Clinical research associates (CRAs) ascertained patients, confirmed eligibility, approached physicians for permission to contact patients, and obtained consent. Patient registration was managed by the CALGB Statistical Center. Patient interviews were completed on the telephone in a similar manner and at comparable time points (mid-treatment and 12 and 24 months) by trained centralized staff for both protocols. Initial interviews lasted $45 \mathrm{~min}$ and follow-up interviews were about 20 min in both studies. Medical records were abstracted by CRAs for tumor and treatment data.

\subsection{Measures}

Type of study was defined by the respective protocol enrollment. This variable is the key variable predicting $\mathrm{QoL}$ outcomes. QoL was measured in both protocols at mid-treatment, 12 and 24 months using the EORTC QLQ-C30 (European Organisation for Research and Treatment of Cancer Quality of Life Questionnaire). ${ }^{9}$ Mid-treatment was on average 3-5 months post-diagnosis in both groups. The EORTC has 30 items from which we evaluated the following subscales: global health status, physical functioning, emotional functioning, cognitive functioning, fatigue, and social functioning. Higher scores on global QoL and the functioning scales indicate better QoL/ function; higher scores on the fatigue scale indicate greater fatigue. We also used the EORTC BR23 (Breast Cancer Module) to measure body image (higher score indicates better image), sexual functioning (higher score indicates better function), and systemic therapy side effects (higher score indicates more problems with side effects).${ }^{10}$ The reliability of these scales, as evaluated with Cronbach's alphas, ranged from 0.60 to 0.89 across the entire sample and was similar across study type (0.63-0.92 for the RCT and $0.60-0.89$ for the observational study; the lowest range of reliability was seen for the emotional and social function scales).

Pre-treatment baseline EORTC-QLQ-C30 was used to control for QoL outcomes in the RCT group. Since the EORTC-QLQ-C30 was not available at baseline (pre-treatment) for the observational study, we used available SF-12 (the 12-Item Short Form Health Survey) measures that were commensurate with corresponding EORTC-QLQ-C30 subscales to control for baseline QoL. For instance, for the EORTC physical functioning scale, we used the SF-12 physical components summary score (PCS) for the observational study participants. Similarly for EORTC emotional and social functioning subscales, we used the SF-12 mental components summary score (MCS); and for the EORTC fatigue symptoms we used the SF-12 item: "How much of the time during the past 4 weeks, did you have a lot of energy?" 
Clinical covariates of QoL outcomes included tumor size, nodal status, ER (estrogen receptor) status, type of surgery (breast conserving vs. mastectomy), and number of comorbidities using the OARS (Older Americans Resources and Services) Multidimensional Functional Assessment 14-item scale. ${ }^{12}$ Setting of care was measured based on type of site (National Cancer Institute designated cancer center vs. other). We did not have data on hormonal therapy, radiation use, or dose intensity in both studies, so could not include these variables in analysis. To control for the effects of social support on QoL, we controlled for emotional/informational support (Cronbach's alpha $=.93$ ) and tangible support (Cronbach's alpha $=.94$ ) using sub-scales of the Medical Outcomes Study (MOS) social support instrument. ${ }^{11}$ Finally, we examined several variables as potential correlates of being treated in the RCT vs. the observational study, including age at enrollment, self-reported race/ ethnicity (categorized as White and non-White), education (< high school or thigh school), and marital status (married vs. single, widowed, divorced, or separated).

\subsection{Statistical Analysis}

We examined univariate associations between type of study (RTC vs. off-trial observational study) and covariates using chi-square tests, the Cochran-Armitage trend test, and t-tests. We report unadjusted- and adjusted-means for QoL scores and 95\% confidence intervals separately for the RCT and the observational study. We used linear mixed-effects modeling to compare changes in adjusted mean QoL scores from mid-treatment to 24 months for women on the RCT vs. the observational study based on all available data. We estimated corresponding contrasts to assess differences in average QoL score changes from midtreatment to 24 months, controlling for available baseline pre-treatment QoL measures and other covariates. The baseline QoL measures (EORTC and SF-12) were standardized as zscores for both RCT and observational study participants to enhance their comparability.

We retained age, education, tumor size, nodes, surgery, comorbidity, social support, and site in the models to capture clinically relevant factors and/or variables that could affect selection into each study; year of enrollment was not significant and was not included in final models. We also performed similar analyses using propensity score matching methods (data not shown). ${ }^{13}$ Since results were consistent across all approaches, we present the results of the linear mixed-effect models for unmatched data. Analyses were performed using SAS 9.2 and the MatchIt package ${ }^{14}$ from R version 2.13.2.

\section{Results}

The response and follow-up rates for the women receiving chemotherapy on- vs. off-trial are shown in Fig. 1. Anthracycline-based regimens were used by 58\% of the women in the RCT and 54\% of those in the observational study; the remainder used CMF-based treatments. The average age of women receiving chemotherapy in these two studies was 71 years (SD 5, range of 65-90 years) (Table 1). Several variables were associated with enrollment in the RCT vs. the observational study and were used to control for differences in QoL outcomes in subsequent analyses (Table 1). For instance, women in the RCT had higher educational levels than women receiving chemotherapy off-trial ( $84 \%$ vs. 54\% high school or greater, $p$ $<.001)$. Of note, women with less tangible support were also more likely to enroll in the $\mathrm{RCT}$, although the absolute average difference was only 5 points on a 100-point scale $(\mathrm{p}=$. 008). Women in the trial also had a greater proportion of larger and/or node positive cancers than those in the observational study.

The trajectories of adjusted QoL over the 24 months after treatment varied between the women who received standard chemotherapy in the RCT and those in the observational study treated with chemotherapy (Fig. 2 and Table 2). Women in the RCT improved to a greater extent relative to their mid-treatment scores than women in the observational study, 
after controlling for baseline QoL, age, education, number of comorbidities, clinical factors and other covariates. For instance, while women in both groups showed what appear to be the beginnings of declines in physical functioning, women in the RCT maintained scores well above their mid-treatment levels (a positive change of 3.3 points) $(95 \% \mathrm{CI}-0.5,7.2)$, while those in the observational study declined to slightly below their mid-treatment level (a change of -1.9 points) $(95 \% \mathrm{CI}-4.4,0.5)(\mathrm{p}=.024$ for the difference) in the 24-month period, controlling for baseline physical function, age, education, number of comorbidities and other covariates (Table 3). A similar pattern and magnitude of difference was seen for all other QoL sub-scales except for sexual and cognitive functioning, where there were smaller differences that did not reach statistical significance.

Women receiving their chemotherapy in the RCT also had significantly greater decreases in treatment-related side effects at 24-months compared to mid-treatment than women in the observational study after adjustment for covariates $(-21.1$ [95\% CI-23.7, -18.6] vs. -8.0 [95\% CI-9.6, -6.3], $\mathrm{p}<.001$ for difference in the mean changes, where a negative sign indicates fewer side effects). In addition, women in the RCT reported less fatigue over time than women in the observational study, controlling for baseline, and pre-treatment fatigue (Table 3).

\section{Discussion}

This is the first study that we are aware of to conduct a planned comparison of the quality of life of older women receiving chemotherapy in an RCT vs. an observational study in the same settings and cared for by the same groups of providers. We found that women who enrolled in the trial had higher educational levels but somewhat lower tangible social support than women who received their chemotherapy in the observational study. After controlling for these and other measured factors including available baseline pre-treatment QoL information, it appears that older women treated in the RCT may have had greater improvements in QoL across almost all domains in the first 24-months post-treatment than women who received similar regimens in the observational study. Women treated in the RCT also appear to have had more improvements in post-treatment symptoms and fatigue than women in the observational study.

The finding that RCT participants had a higher educational level than those in the observational study is not surprising and suggests that recruitment and RCT consent materials for older patients may need to consider literacy. ${ }^{15,16}$ While the absolute difference in tangible support between the groups was only 5 points, the result that women with lower tangible social support were significantly more likely to enroll in the trial than the observational study is intriguing. It is possible that women with low support recognized that the RCT structure could provide resources to facilitate their receipt of chemotherapy. If corroborated, this could be a potential leverage point for increasing participation of older women in clinical trials.

The finding that QoL improved in both groups over the first 12 months post-treatment is similar to the trajectory seen in younger breast cancer patients. ${ }^{17}$ However, in contrast to younger patients, our older patients seemed to experience some declines in QoL from 12 to 24 months post-treatment. While not statistically significant, if this is confirmed, it might suggest that age-related declines may begin to overshadow initial improvements after therapy. Alternatively, the older age group may be experiencing decrements in QoL related to late- or delayed-effects of treatment based on interactions of chemotherapy and comorbid conditions. ${ }^{17}$ These will be important areas for further research and examination of trends over longer periods of time. 
We found that for almost all domains of QoL, including global QoL, physical, emotional and social function, fatigue, body image and side effects, chemotherapy-treated older breast cancer patients in the RCT had greater improvements by 24 months than those in the observational study, even after considering pre-treatment QoL and their lower mid-treatment levels. Moreover, the improvements observed 24 months post-chemotherapy for the women in the RCT would be considered clinically relevant and in the "medium range" based on a change of approximately 10 points on the EORTC. ${ }^{18-20}$ This magnitude was seen for global QoL and several sub-domains. The differences for cognitive functioning were not statistically significant, but this sub-scale has been reported to have a much narrower range within which to detect meaningful differences. ${ }^{20}$ Also, patients in both studies were prescreened for cognitive deficits in determining eligibility, limiting heterogeneity in cognitive functioning and ability to detect group differences.

Since prior assessments of meaningful differences on the EORTC have been conducted among patients who were almost all under age $65,{ }^{18-20}$ it is possible that smaller changes might translate into important differences in ability of older women to maintain independent living and function, since older women already have more limitations prior to cancer than younger women. ${ }^{21,22}$ Overall, while our results will need to be confirmed, the differences in adjusted mean score changes over time for the RCT vs. the observational study suggest that the women in the RCT may have experienced clinically meaningfully better QoL outcomes than women receiving the same therapies outside of a trial setting and enrolled in an observational study.

There are several credible explanations for the significantly better QoL changes reported by women who received standard therapy in a trial compared to their counterparts who received the same therapy in the same sites by the same groups of providers in the observational study. ${ }^{23}$ First, it is possible that the monitoring required in the trial detected medical conditions that could be treated, improving QoL. Next, the added monitoring by RCT staff could have resulted in better symptom management than occurred in routine practice. Alternatively, the added attention and intensity of monitoring in the RCT may have improved access to the treatment team, enhanced patient-physician communication and provided a greater sense of patient well-being. Another alternative explanation is that while both groups had comparable rates of chemotherapy regimens, the RCT group may have received treatment that was more adherent to guidelines (e.g., more intensive doses, greater supportive care to complete planned cycles) than the observational group, and thus had lower mid-treatment QoL values as seen in the RCT vs. the observational group, after considering stage differences. The improvements over time for the RCT group could then represent regression to the mean, or the fact that trial monitoring was sufficient to counter any initial deleterious effects of more intensive trial-based treatment. Since we do not have data to determine which, if any, of these factors mediate the observed differences in QoL changes between the trial and off-trial groups, this will be an important area for future research if our results are confirmed.

While we cannot rule out unmeasured baseline differences between the two study groups, the studies were designed in parallel for this planned comparison with comparable eligibility criteria and the outcome differences were robust after controlling for the variables that are traditionally related to both selection biases and QoL outcomes, such as pre-treatment QoL, age, education, and comorbidity. The results for the 4 scales where we had pre-treatment QoL were virtually unchanged whether they were adjusted or unadjusted for baseline pretreatment QoL (not shown), suggesting that pre-treatment differences in the groups were unlikely to account for the differences in 24-month outcomes observed. While our conclusion has face validity, prior studies comparing outcomes in RCT vs. non-RCT groups have been inconsistent. ${ }^{23,24}$ Thus, our results will need to be confirmed using a clinical trial 
design, where receipt of treatment on- vs. off-trial is randomly allocated to eliminate any unmeasured confounding variables associated with imbalances in the groups and study selection. ${ }^{23}$ If confirmed, it will also be important to conduct future research to understand the mechanisms that underlie different QoL trajectories.

There are several other caveats that should be considered in evaluating our results. The average QoL scores in these two studies may not be representative of all older breast cancer patients receiving chemotherapy since research volunteers tend to be healthier than average. ${ }^{25,26}$ However, this should not affect the internal validity of the QoL comparisons. The two studies had different rates of lost to follow-up, with higher rates in the observational study, although some of the women treated off-trial were not yet due for follow-up at the time of this analysis. Higher retention in the RCT may reflect the greater involvement of women in the RCT vs. the observational study, since one was a treatment study and the other was not. However, there were no systematic differences in correlates of follow-up losses between the studies (not shown), so comparisons of QoL are likely to be internally valid. Finally, we did not have detailed and/or comparable treatment information on factors like drug doses, symptom management and support, types of axillary procedures, and only had information on hormonal therapy for the observational study and radiation for the RCT. Thus, we cannot rule out unmeasured confounding variables of QoL outcomes due to treatment variations. However, it is not likely that the RCT patients had lower levels of use of radiation or hormonal therapy or less dose intensity than the observational group explaining their better QoL outcomes.

In summary, these results are suggestive that there could be different QoL trajectories for older women undergoing standard chemotherapy on- vs. off-trial. These results need to be confirmed. If verified in future research and with other disease groups, it is possible that the extra monitoring and reporting within a clinical trial might provide the infrastructure for interventions to address symptoms and improve quality of life among the growing older cancer population.

\section{Acknowledgments}

We gratefully acknowledge the women who participated in these studies and shared so generously their time and experiences. In addition, we acknowledge the clinical research associates at the study sites who enrolled patients and submitted clinical data; without them the study would not have been possible. We acknowledge Dr. Alice Kornblith for her leadership of the quality of life sub-study of \#49907 and Dr. Richard Schilsky for his support of this research.

We acknowledge Wanda Lucas, Trina McClendon and Meghan McGuckin for their work in interviewing the patients for the observational study. We also acknowledge Edie Fitts, advocate, the CALGB Statistical Center (John Postiglione, Peggy Edwards and Lydia Hodgson) and CALGB Central Office staff who supported these protocols (Kathy Karas, Sara Hoffman, Mary Sherrell, Avis Rodgers and Donna Johnson). We are grateful to the Principal Investigators for protocol \#369901 and \#49907 at the CALGB sites for facilitating patient recruitment.

\section{REFERENCES}

1. Surveillance, Epidemiology, and End Results (SEER) Program SEER*Stat Database, National Cancer Institute, DCCPS, Surveillance Research Program, Surveillance Systems Branch, released April 2012. [accessed May 1 2012] www.seer.cancer.gov

2. Mitka M. Too few older patients in cancer trials: experts say disparity affects research results and care. JAMA. 2003; 290:27-28. [PubMed: 12837701]

3. Kemeny MM, Peterson BL, Kornblith AB, et al. Barriers to clinical trial participation by older women with breast cancer. J Clin Oncol. 2003; 21:2268-2275. [PubMed: 12805325]

4. Muss HB, Berry DA, Cirrincione CT, et al. Adjuvant chemotherapy in older women with earlystage breast cancer. N Engl J Med. 2009; 360:2055-2065. [PubMed: 19439741] 
5. Mandelblatt JS, Sheppard VB, Hurria A, et al. Breast cancer adjuvant chemotherapy decisions in older women: the role of patient preference and interactions with physicians. J Clin Oncol. 2010; 28:3146-3153. [PubMed: 20516438]

6. Kornblith AB, Lan L, Archer L, et al. Quality of life of older patients with early-stage breast cancer receiving adjuvant chemotherapy: a companion study to cancer and leukemia group B 49907. J Clin Oncol. 2011; 29:1022-1028. [PubMed: 21300923]

7. Mandelblatt JS, Faul LA, Luta G, et al. Patient and physician decision syles and breast cancer chemotherapy use in older women: Cancer and Leukemia Group B Protocol \#369901. J Clin Oncol. 2013 [in press].

8. Kawas C, Karagiozis H, Resau L, et al. Reliability of the Blessed Telephone Information-MemoryConcentration Test. J Geriatr Psychiatry Neurol. 1995; 8:238-242. [PubMed: 8561839]

9. Aaronson, N., et al. The quality of life of cancer patients, in multidimensional approach to the measurement of quality of life in lung cancer clinical trials. Aaronson, Beckmann J., editor. New York: Raven Press; 1987. p. 63-82.

10. Sprangers MAG, Groenvold M, Arraras JI, et al. The European organization for research and treatment of cancer breast cancer-specific quality-of-life questionnaire module: first results from a three-country field study. J Clin Oncol. 1996; 14:2756-2768. [PubMed: 8874337]

11. Sherbourne CD, Stewart AL. The MOS social support survey. Soc Sci Med. 1991; 32:705-714. [PubMed: 2035047]

12. Fillenbaum GG, Smyer MA. The development, validity, and reliability of the OARS multidimensional functional assessment questionnaire. J Gerontol. 1981; 36:428-434. [PubMed: 7252074]

13. Stuart EA. Matching methods for causal inference: a review and a look forward. Stat Sci. 2010; 25:1-21. [PubMed: 20871802]

14. Ho D, Imai K, King G, et al. MatchIt: Nonparametric preprocessing for parametric causal inference. J Stat Softw. 2011; 42:1-28.

15. Miller SM, Hudson SV, Egleston BL, et al. The relationships among knowledge, self-efficacy, preparedness, decisional conflict, and decisions to participate in a cancer clinical trial. Psychooncology Feb. 2012; 14 http://dx.doi.org/10.1002/pon.3043[Epub ahead of print].

16. Knapp P, Mitchell N, Raynor DK, et al. Can we improve recruitment to trials and informed consent by improving participant information sheets? Trials. Dec 13.2011 12(Suppl 1):A123. [Epub ahead of print].

17. Jeffe DB, Perez M, Liu Y, et al. Quality of life over time in women diagnosed with ductal carcinoma in situ, early-stage invasive breast cancer, and age-matched controls. Breast Cancer Res Treat. Apr 8.2012 [Epub ahead of print].

18. Osoba D, Rodrigues G, Myles J, et al. Interpreting the significance of changes in health-related quality-of-life scores. J Clin Oncol. 1998; 16:139-144. [PubMed: 9440735]

19. King MT. The interpretation of scores from the EORTC quality of life questionnaire QLQ-C30. Qual Life Res. 1996; 5:555-567. [PubMed: 8993101]

20. Cocks K, King MT, Velikova G, et al. Evidence-based guidelines for determination of sample size and interpretation of the European Organisation for the Research and Treatment of Cancer Quality of Life Questionnaire Core 30. J Clin Oncol. 2011; 29:89-96. [PubMed: 21098316]

21. Silliman RA, Prout MN, Field T, et al. Risk factors for a decline in upper body function following treatment for early stage breast cancer. Breast Cancer Res Treat. 1999; 54:25-30. [PubMed: 10369077]

22. Mandelblatt J, Edge S, Meropol N, et al. Sequelae of axillary lymph node dissection in older women with stage 1 and 2 breast carcinoma. Cancer. 2002; 95:2445-2454. [PubMed: 12467056]

23. Peppercorn JM, Weeks JC, Cook EF, Joffe S. Comparison of outcomes in cancer patients treated within and outside clinical trials: conceptual framework and structured review. Lancet. Jan 24; 2004 363(9405):263-270. [PubMed: 14751698]

24. Vist GE, Bryant D, Somerville L, Birminghem T, Oxman AD. Outcomes of patients who participate in randomized controlled trials compared to similar patients receiving similar interventions who do not participate. Cochrane Database Syst Rev Jul. 2008; 16(3):MR000009. http://dx.doi.org/10.1002/14651858.MR000009.pub4. 
25. Almeida L, Kashdan TB, Nunes T, et al. Who volunteers for phase I clinical trials? Influences of anxiety, social anxiety and depressive symptoms on self-selection and the reporting of adverse events. Eur J Clin Pharmacol. Jun; 2008 64(6):575-582. [PubMed: 18320183]

26. Pinsky PF, Miller A, Kramer BS, et al. Evidence of a healthy volunteer effect in the prostate, lung, colorectal, and ovarian cancer screening trial. Am J Epidemiol. 2007; 165(8):874-881. [PubMed: 17244633] 


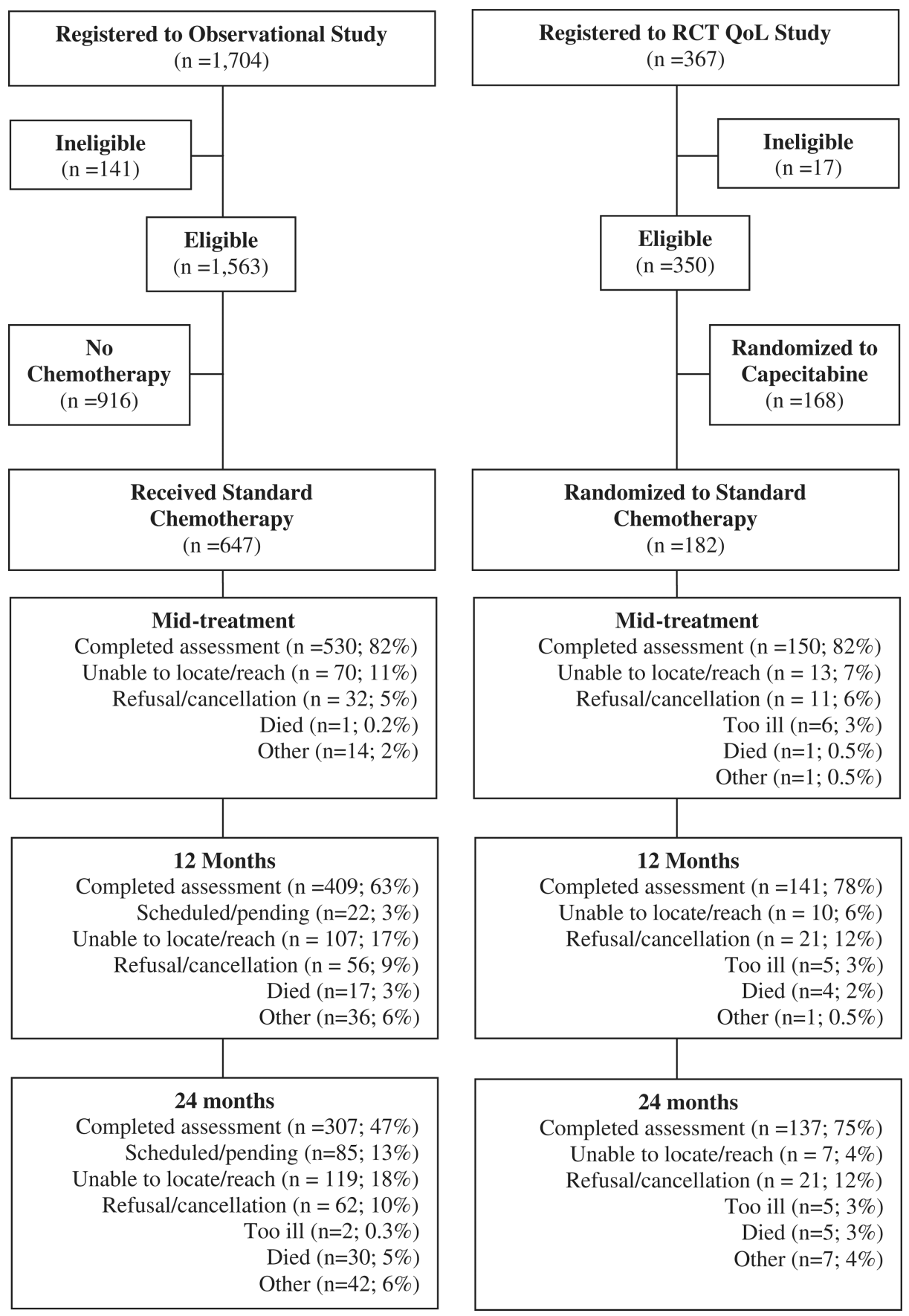

Fig. 1.

Study sample of older breast cancer patients enrolled in a randomized clinical trial vs. an observational study. 

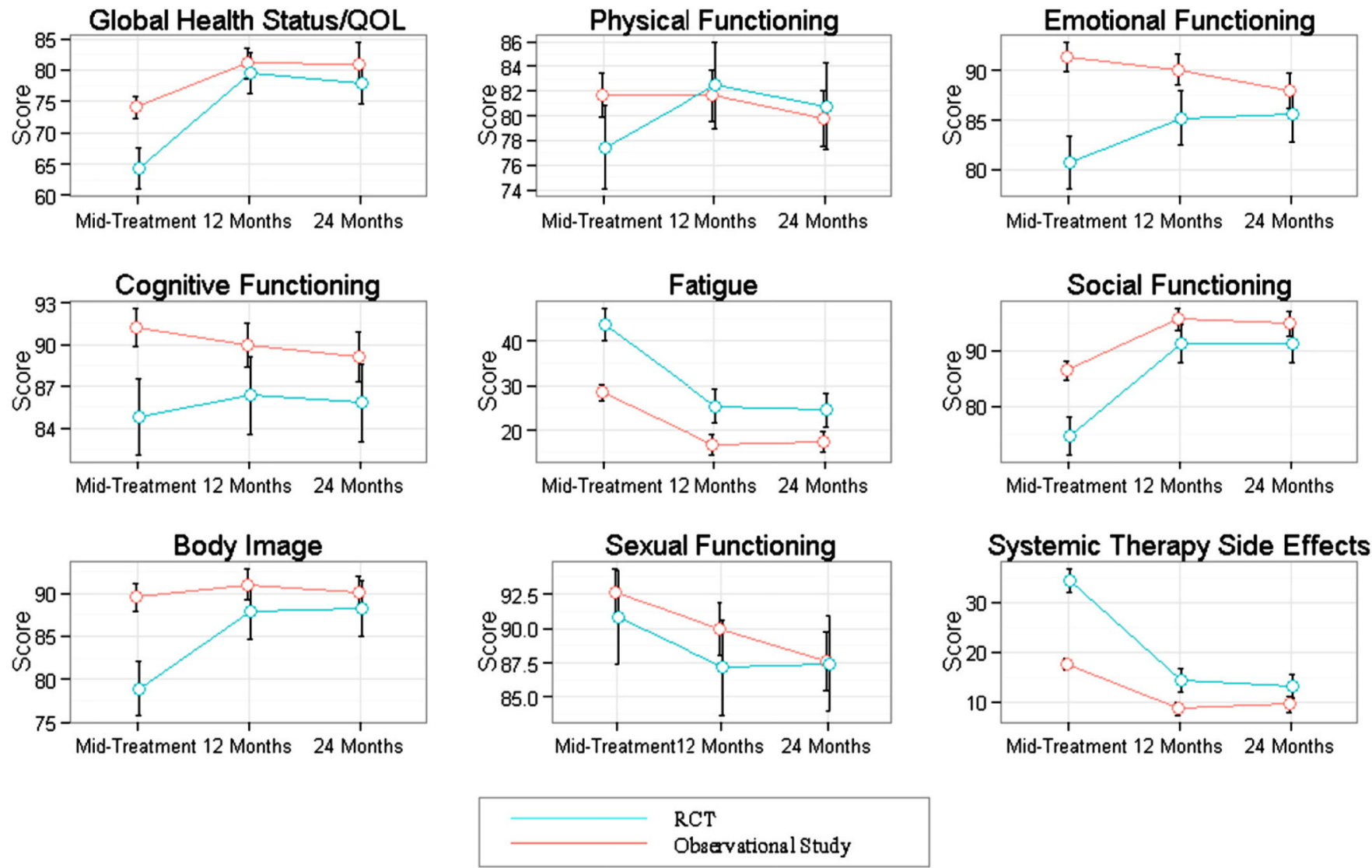

Fig. 2.

Trajectories of adjusted mean quality of life (QoL) scores among older breast cancer patients receiving chemotherapy in a randomized clinical trial vs. an observational study. 
Table 1

Characteristics of older breast cancer patients getting chemotherapy on- vs. off-trial.

\begin{tabular}{|c|c|c|c|c|}
\hline Age at enrollment, mean (SD) & $\frac{\text { All }(n=712)}{71.1(\text { SD 4.9) }}$ & $\frac{\operatorname{RCT}(n=182)^{a}}{72.0(\operatorname{SD} 4.6)}$ & $\frac{\text { Off-trial Observational Study }(n=530)^{b}}{70.8(\text { SD 5.0) }}$ & $\frac{p \text {-value }}{0.002}$ \\
\hline \multicolumn{5}{|l|}{ Race, n (\%) } \\
\hline White, non-Hispanic & $615(86 \%)$ & $157(86 \%)$ & $458(86 \%)$ & 0.959 \\
\hline Non-white & $97(14)$ & $25(14)$ & $72(14)$ & \\
\hline \multicolumn{5}{|l|}{ Education level, n (\%) } \\
\hline High school grad & $437(61)$ & $153(84)$ & $284(54)$ & $<.001$ \\
\hline$<$ High school grad & $245(34)$ & $16(9)$ & $229(43)$ & \\
\hline Missing & $30(4)$ & $13(7)$ & $17(3)$ & \\
\hline \multicolumn{5}{|l|}{ Marital status, n (\%) } \\
\hline Married & $414(58)$ & $95(52)$ & $319(60)$ & 0.320 \\
\hline Not married & $282(40)$ & $74(41)$ & $208(39)$ & \\
\hline Missing & $16(2)$ & $13(7)$ & $3(1)$ & \\
\hline \multicolumn{5}{|l|}{ ER status, n (\%) } \\
\hline Positive & $239(34)$ & $65(36)$ & $174(33)$ & 0.497 \\
\hline Negative & $471(66)$ & $117(64)$ & $354(67)$ & \\
\hline Missing & $2(0)$ & $0(0)$ & $2(0)$ & \\
\hline \multicolumn{5}{|l|}{ Tumor size, n (\%) } \\
\hline$<2 \mathrm{~cm}$ & $297(42)$ & $67(37)$ & $230(43)$ & 0.005 \\
\hline 2 to $<3 \mathrm{~cm}$ & $198(28)$ & $42(23)$ & $156(29)$ & \\
\hline $3+\mathrm{cm}$ & $215(30)$ & $72(40)$ & $143(27)$ & \\
\hline Missing & $2(0)$ & $1(1)$ & $1(0)$ & \\
\hline \multicolumn{5}{|l|}{ Nodal status, n (\%) } \\
\hline Node positive & $446(63)$ & $128(70)$ & $318(60)$ & 0.012 \\
\hline Node negative & $263(37)$ & $53(29)$ & $210(40)$ & \\
\hline Missing & $3(0)$ & $1(1)$ & $2(0)$ & \\
\hline \multicolumn{5}{|l|}{ Surgery, n (\%) } \\
\hline Breast conserving & $381(54)$ & $77(42)$ & $304(57)$ & $<.001$ \\
\hline Mastectomy & $328(46)$ & $104(57)$ & $224(42)$ & \\
\hline Missing & $3(0)$ & $1(1)$ & $2(0)$ & \\
\hline Emotional support, mean (SD) & $85.5(17.8)$ & $84.2(19.3)$ & $85.9(17.3)$ & 0.313 \\
\hline Tangible support, mean (SD) & $86.8(19.6)$ & $83.1(21.3)$ & $88.0(18.9)$ & 0.008 \\
\hline \multicolumn{5}{|l|}{ \# Comorbidities, n (\%) } \\
\hline 0 & $51(7)$ & $19(10)$ & $32(6)$ & 0.0164 \\
\hline 1 & $138(19)$ & $40(22)$ & $98(18)$ & \\
\hline 2 & $178(25)$ & $40(22)$ & $138(26)$ & \\
\hline $3+$ & $326(46)$ & $70(38)$ & $256(48)$ & \\
\hline Missing & $19(3)$ & $13(7)$ & $6(1)$ & \\
\hline \multicolumn{5}{|l|}{ Type of site, $\mathrm{n}(\%)$} \\
\hline Comprehensive cancer center & 237 (33) & $74(41)$ & $163(31)$ & 0.014 \\
\hline
\end{tabular}




\begin{tabular}{|c|c|c|c|c|}
\hline & All $(n=712)$ & $\underline{\operatorname{RCT}(n=182)^{a}}$ & Off-trial Observational Study $(n=530)^{b}$ & $\underline{p \text {-value }}{ }^{c}$ \\
\hline Age at enrollment, mean (SD) & 71.1 (SD 4.9) & 72.0 (SD 4.6) & 70.8 (SD 5.0) & 0.002 \\
\hline Community site & $475(67)$ & $108(59)$ & $367(69)$ & \\
\hline
\end{tabular}

Legend: RCT: Randomized clinical trial, SD: Standard deviation, ER: Estrogen receptor.

${ }^{a}$ There were 182 women completing intake interviews on the RCT; of these 150 completed mid-treatment interviews and are included in subsequent analyses.

$b$

${ }^{b}$ There were 647 women registered to the observational study who received chemotherapy; 530 of these women completed intake interviews. Intake is at mid-treatment for the observational study.

${ }^{c} P$-values from Student's t-tests comparing means, chi-square tests comparing categorical counts, and Cochran-Armitage trend test for comparing the number of comorbidities between groups. 
Table 2

Unadjusted and adjusted ${ }^{a}$ means of quality of life scores from mid-treatment to 24 months.

\begin{tabular}{|c|c|c|c|c|}
\hline \multirow{3}{*}{ Measure } & \multicolumn{2}{|c|}{$\operatorname{RCT}(\mathbf{n}=150)$} & \multicolumn{2}{|c|}{ Observational study $(n=530)$} \\
\hline & Unadjusted & Adjusted & Unadjusted & Adjusted \\
\hline & \multicolumn{2}{|c|}{ Mean $(95 \%$ CI $)$} & \multicolumn{2}{|c|}{ Mean $(95 \%$ CI $)$} \\
\hline \multicolumn{5}{|c|}{ Global health status/QoL } \\
\hline Mid-treatment & $64.0(60.4,67.6)$ & $64.3(61.0,67.6)$ & $73.7(72.0,75.4)$ & $74.1(72.4,75.8)$ \\
\hline 12 months & $79.2(76.5,81.8)$ & $79.5(76.1,82.9)$ & $81.8(79.5,84.1)$ & $81.1(78.6,83.6)$ \\
\hline 24 months & $77.2(74.3,80.1)$ & $77.9(74.5,81.3)$ & $82.3(79.4,85.3)$ & $81.0(77.7,84.4)$ \\
\hline \multicolumn{5}{|c|}{ Physical functioning } \\
\hline Mid-treatment & $77.0(73.9,80.1)$ & $77.4(74.0,80.8)$ & $80.2(78.2,82.3)$ & $81.7(79.9,83.5)$ \\
\hline 12 months & $82.1(79.2,85.1)$ & $82.5(79.0,85.9)$ & $81.6(79.3,83.8)$ & $81.6(79.6,83.7)$ \\
\hline 24 months & $80.2(77.1,83.4)$ & $80.7(77.2,84.3)$ & $80.7(78.2,83.1)$ & $79.8(77.5,82.0)$ \\
\hline \multicolumn{5}{|c|}{ Emotional functioning } \\
\hline Mid-treatment & $80.7(77.5,83.9)$ & $80.7(78.1,83.4)$ & $91.3(90.1,92.5)$ & $91.4(89.9,92.8)$ \\
\hline 12 months & $84.8(82.0,87.6)$ & $85.2(82.5,87.9)$ & $90.7(89.3,92.1)$ & $90.1(88.5,91.7)$ \\
\hline 24 months & $84.7(81.7,87.7)$ & $85.6(82.8,88.3)$ & $88.8(86.9,90.7)$ & $87.9(86.2,89.7)$ \\
\hline \multicolumn{5}{|c|}{ Cognitive functioning } \\
\hline Mid-treatment & $83.8(80.7,86.9)$ & $84.9(82.1,87.6)$ & $90.8(89.5,92.1)$ & $91.2(89.8,92.6)$ \\
\hline 12 months & $85.2(83.0,87.5)$ & $86.4(83.6,89.1)$ & $90.3(88.8,91.8)$ & $90.0(88.4,91.6)$ \\
\hline 24 months & $84.7(82.2,87.2)$ & $85.8(83.1,88.6)$ & $89.3(87.6,91.0)$ & $89.1(87.4,90.9)$ \\
\hline \multicolumn{5}{|l|}{ Fatigue } \\
\hline Mid-treatment & $43.2(39.2,47.2)$ & $43.8(40.1,47.5)$ & $28.9(26.8,31.0)$ & $28.5(26.6,30.4)$ \\
\hline 12 months & $25.5(22.6,28.4)$ & $25.4(21.7,29.2)$ & $16.9(15.1,18.8)$ & $16.7(14.6,18.9)$ \\
\hline 24 months & $24.6(21.6,27.6)$ & $24.5(20.8,28.3)$ & $17.9(15.6,20.2)$ & $17.4(15.0,19.8)$ \\
\hline \multicolumn{5}{|c|}{ Social functioning } \\
\hline Mid-treatment & $75.3(71.0,79.6)$ & $74.7(71.3,78.0)$ & $86.8(84.9,88.7)$ & $86.5(84.7,88.2)$ \\
\hline 12 months & $91.4(88.5,94.3)$ & $91.1(87.7,94.6)$ & $95.3(94.0,96.6)$ & $95.7(93.7,97.7)$ \\
\hline 24 months & $91.2(88.1,94.3)$ & $91.3(87.8,94.7)$ & $95.1(93.5,96.8)$ & $94.9(92.7,97.1)$ \\
\hline \multicolumn{5}{|c|}{ Sexual functioning } \\
\hline Mid-treatment & $90.8(87.6,94.0)$ & $90.8(87.4,94.3)$ & $91.9(90.4,93.3)$ & $92.6(90.8,94.3)$ \\
\hline 12 months & $87.1(83.3,90.9)$ & $87.1(83.7,90.6)$ & $90.1(88.4,91.8)$ & $89.9(88.0,91.9)$ \\
\hline 24 months & $87.8(84.0,91.5)$ & $87.4(84.0,90.9)$ & $87.9(85.8,90.1)$ & $87.6(85.5,89.7)$ \\
\hline \multicolumn{5}{|l|}{ Body image } \\
\hline Mid-treatment & $78.6(75.0,82.2)$ & $79.0(75.8,82.1)$ & $90.0(88.4,91.5)$ & $89.6(87.9,91.2)$ \\
\hline 12 months & $87.2(84.4,89.9)$ & $87.8(84.6,91.0)$ & $91.3(89.6,92.9)$ & $91.0(89.2,92.8)$ \\
\hline 24 months & $87.5(84.6,90.4)$ & $88.3(85.1,91.5)$ & $90.6(88.7,92.4)$ & $90.0(88.1,92.0)$ \\
\hline \multicolumn{5}{|l|}{$\begin{array}{l}\text { Systemic therapy } \\
\text { side effects }\end{array}$} \\
\hline Mid-treatment & $33.5(31.0,36.0)$ & $34.3(32.1,36.6)$ & $17.9(16.5,19.3)$ & $17.5(16.3,18.7)$ \\
\hline 12 months & $13.9(11.8,15.9)$ & $14.4(12.1,16.8)$ & $8.6(7.7,9.5)$ & $8.7(7.3,10.1)$ \\
\hline 24 months & $13.2(11.1,15.2)$ & $13.2(10.8,15.6)$ & $9.7(8.5,10.9)$ & $9.5(8.0,11.1)$ \\
\hline
\end{tabular}


Legend: RCT: Randomized clinical trial, QoL: Quality of life, CI: Confidence interval.

${ }^{a}$ Adjusted for age, education, tumor size, nodes, surgery, comorbidity, tangible support, and site type; adjusted for baseline pre-treatment physical, emotional, social function and fatigue. We did not have comparable data on hormonal or radiation therapy or types of axillary procedures, baseline body image, sexual or cognitive function across the studies so these are not included in the analysis. 
Table 3

Adjusted mean changes from mid-treatment to 24 months for quality of life scores among older women receiving chemotherapy in an RCT vs. an observational study ${ }^{a}$.

\begin{tabular}{|lcccc|}
\hline Measure $^{b}$ & $\begin{array}{c}\text { RCT } \\
\mathbf{N = 1 5 0}\end{array}$ & $\begin{array}{c}\text { Observational study } \\
(\mathbf{n}=\mathbf{5 3 0})\end{array}$ & $\begin{array}{c}\text { Difference } \\
\text { (RCT-observational) }\end{array}$ & P \\
\hline Global health status/QoL & $13.7(10.2,17.1)$ & $7.0(3.5,10.4)$ & $6.7(1.8,11.6)$ & 0.007 \\
Physical functioning & $3.3(-0.5,7.2)$ & $-1.9(-4.4,0.5)$ & $5.3(0.7,9.8)$ & 0.024 \\
Emotional functioning & $4.9(2.0,7.8)$ & $-3.4(-5.3,-1.6)$ & $8.3(4.8,11.8)$ & $<.001$ \\
Cognitive functioning & $1.0(-2.0,4.0)$ & $-2.1(-4.0,-0.2)$ & $3.1(-0.5,6.6)$ & 0.090 \\
Fatigue & $19.2(-23.7,-14.8)$ & $-11.1(-13.8,-8.3)$ & $-8.1(-13.4,-2.9)$ & 0.002 \\
Social functioning & $16.6(12.7,20.4)$ & $8.4(6.0,10.9)$ & $8.2(3.6,12.7)$ & $<.001$ \\
Sexual functioning & $-3.4(-6.6,-0.2)$ & $-5.0(-6.9,-3.0)$ & $1.6(-2.2,5.4)$ & 0.405 \\
Body image & $9.3(6.4,12.3)$ & $0.5(-1.4,2.4)$ & $8.8(5.3,12.4)$ & $<.001$ \\
Systemic therapy side effects & $-21.1(-23.7,-18.6)$ & $-8.0(-9.6,-6.3)$ & $-13.2(-16.2,-10.1)$ & $<.001$ \\
\hline
\end{tabular}

We did not have comparable data on hormonal or radiation therapy or types of axillary procedures, baseline body image, sexual or cognitive function across the studies, so these are not included in the analysis.

Legend: RCT: Randomized clinical trial, QoL: Quality of life.

${ }^{a}$ Linear mixed effects models, controlling for age, education, tumor size, nodal status, surgery type, number of comorbidities, tangible social support, and site type; models for physical functioning, emotional functioning, social functioning, and fatigue outcomes included a covariate for baseline pre-treatment level of these measures to control for potential baseline differences.

${ }^{b}$ For global QoL, physical functioning, emotional functioning, cognitive functioning, social functioning, sexual functioning and body image, a higher score indicates better function. For fatigue and side effects, a higher score indicates greater problems. So, a reduction in these scores (negative sign), indicates an improvement, or decline in problems. 